\title{
FORMALISMS OF THE COMPLEX IMPEDANCE SPECTROSCOPY
}

\author{
Open Engineering Collaboration*†
}

November 24, 2020

\begin{abstract}
The Complex Impedance Spectroscopy (CIS) technique is used to characterize materials with electrical and ferroelectric properties. In this paper, we present the 4 formalisms for analyzing the data obtained by the CIS.
\end{abstract}

keywords: complex impedance spectroscopy, materials characterization, complex impedance, complex electrical module, complex dielectric permittivity, complex admittance.

\section{Introduction}

1. Ferroelectric ceramics have properties that are investigated by the non-destructive technique called Complex Impedance Spectroscopy (CIS).

2. There are 4 formalities for analyzing CIS data, namely: Complex Impedance $\left(Z^{*}\right)$, Complex Electrical Module $\left(M^{*}\right)$, Complex Dielectric Permittivity $\left(\varepsilon^{*}\right)$, and Complex Admittance $\left(Y^{*}\right)[1,2]$.

*All authors with their affiliations appear at the end of this paper.

†Corresponding author: andre@selfchargebox.com | Open Engineering Collaboration. 


\section{Methods}

3. A systematic literature review was carried out in the area of materials science and engineering.

\section{Results and Discussion}

4. First formality for analyzing CIS data: Complex Impedance formalism $\left(Z^{*}\right)[3]$.

5. $Z^{*}$ is the ratio of electrical current (Eq. 6) to electrical voltage (Eq. 7), where $\theta_{0}$ is phase angle between $i(t)$ and $V(t)$.

6. $i(t)=I_{m} \sin \left(\omega t+\theta_{0}\right)$

7. $V(t)=V_{m} \sin \left(\omega t+\theta_{0}\right)$

8. If $\theta_{0}=0$, then $Z^{*}$ is purely resistive and does not depend on the frequency:

9. $Z^{\prime}=\frac{V(t)}{i(t)}$

10. If $\theta_{0} \neq 0$, then $Z^{*}$ has resistive and reactive properties, similar to an $\mathrm{RC}$ circuit (resistor-capacitor circuit):

11. $Z^{*}(\omega)=\frac{R}{[1+(\omega \tau)]}-j \frac{R \omega \tau}{\left[1+(\omega \tau)^{2}\right]}=Z^{\prime}(\omega)+j Z^{\prime \prime}(\omega)$

12. Second formality for analyzing CIS data: Complex Dielectric Permittivity formalism $\left(\varepsilon^{*}\right)$ 4, 5].

13. $\varepsilon^{*}=\epsilon^{\prime}+i \varepsilon^{\prime \prime}$

14. $\varepsilon^{\prime}$ of materials with thickness $(d)$ and area $(A)$ is described by:

15. $\varepsilon^{\prime}=\frac{d Z^{\prime \prime}}{\omega \varepsilon_{0} A\left|Z^{*}\right|^{2}}$

16. $\varepsilon^{\prime \prime}$ of materials with thickness $(d)$ and area $(A)$ is described by: 
17. $\varepsilon^{\prime \prime}=\frac{d Z^{\prime}}{\omega \varepsilon_{0} A\left|Z^{*}\right|^{2}}$

18. The ratio between (17) and (15) is called dielectric loss and is related to heat dissipation.

19. $\tan (\delta)=\frac{\varepsilon^{\prime \prime}}{\varepsilon^{\prime}}$

20. Third formality for analyzing CIS data: Electrical Module formalism $\left(M^{*}\right)$ [6,7].

21. $M^{*}$ is a function of the material's capacitance $\left(C_{0}\right)$ :

22. $M^{*}=j \omega C_{0} Z^{*}$

23. Fourth formality for analyzing CIS data: Complex Admittance formalism $\left(Y^{*}\right) \mid 8$.

24. $Y^{*}$ is defined as the inverse of $Z^{*}$. $Y^{\prime}$ is called conductance and $Y^{\prime \prime}$ is susceptibility.

25. $Y^{*}=Y^{\prime}+Y^{\prime \prime}=\frac{1}{Z^{*}}$

\section{Final Remarks}

26. The 4 formalisms are listed according to Table 1, where $\mu$ is the interrelation factor $\mu=j \omega C_{0}$.

\begin{tabular}{cc|cccc}
\hline & $\mathrm{M}$ & $\mathrm{Z}$ & $\mathrm{Y}$ & $\varepsilon$ \\
\hline $\mathrm{M}$ & $\mathrm{M}$ & $\mu Z$ & $\mu Y^{-1}$ & $\varepsilon^{-1}$ \\
$\mathrm{Z}$ & $\mu^{-1} M$ & $\mathrm{Z}$ & $\mathrm{Y}^{-1}$ & $\mu^{-1} \varepsilon^{-1}$ \\
$\mathrm{Y}$ & $\mu M^{-1}$ & $Z^{-1}$ & $\mathrm{Y}$ & $\mu \varepsilon$ \\
$\varepsilon$ & $M^{-1}$ & $\mu^{-1} Z^{-1}$ & $\mu^{-1} Y$ & $\varepsilon$ \\
\hline \hline
\end{tabular}

Table 1: Relationship between formalities of the CIS technique [9]. 


\section{Open Invitation}

Review, add content, and co-author this paper $[10 \mid$. Join the Open Engineering Collaboration.

Send your contribution to andre@selfchargebox.com

\section{Agreement}

All authors agree with [11.

\section{References}

[1] Macdonald, J. R., "Some new directions in impedance spectroscopy data analysis." Electrochimica Acta, 09 Oct. 2020. https://doi.org/10.1016/0013-4686(93)80310-V

[2] Macdonald, J. R., "Impedance spectroscopy: old problems and new developments." Electrochimica Acta, 11 Oct. 2020. https://doi.org/10.1016/0013-4686(90)80002-6

[3] Essaleh, L., et al., "Analysis of complex impedance of p-CuIn3Se5 by impedance spectroscopy." Journal of Alloys and Compounds, 10 Oct. 2020 https://doi.org/10.1016/j.jallcom.2016.07.183

[4] Serrano, A. G., et al., "Microstructural, structural and electrical properties of bilayered BaZr0.05Ti0.95O3/Ba0.75 Sr0.25TiO3 ceramics." Materials Research Bulletin, 10 Oct. 2020. https://doi.org/10.1016/j.materresbull.2016.11.016

[5] Lu, Z. G., "Frequency dependence of the complex dielectric permittivity of ferroelectric relaxors." Physical Review B, 11 Oct. 2020 https://doi.org/10.1103/PhysRevB.51.2694 
[6] Almond, D. P. and West, A.R., "Impedance and modulus spectroscopy of "real" dispersive conductors." Solid State Ionics, 10 Oct. 2020. https://doi.org/10.1016/0167-2738(83)90063-2

[7] Ranjana, R., et al., "Impedance and electric modulus analysis of Sm-modified $\mathrm{Pb}(\mathrm{Zr} 0.55 \mathrm{Ti} 0.45) 1 \mathrm{x} / 4 \mathrm{O} 3$ ceramics." Journal of Alloys and Compounds, 12 Oct. 2020. https://doi.org/10.1016/j.jallcom.2011.03.003

[8] Burgess, A. S. and Cole, P. H., "Design of Acoustic SurfaceWave Devices Using an Admittance Formalism." IEEE Transactions on Microwave Theory and Techniques, 16 Oct. 2020. https://doi.org/10.1109/TMTT.1973.1128090

[9] Barsoukov, E. and Macdonald, J. R., "Impedance spectroscopy: Theory, experiment, and applications." 2nd. ed. [S.1.]: John Wiley Sons, 2005.

[10] Bonaventura, A. L., et al., "Formalisms of the Complex Impedance Spectroscopy." $\quad$ OSF, 20 Oct. 2020. https://doi.org/10.31219/osf.io/2mtk7

[11] Lobo, Matheus P. "Open Journal of Mathematics and Physics (OJMP)." OSF, 21 Oct. 2020. https://doi.org/10.17605/osf.io/6hzyp

\section{The Open Engineering Collaboration}

André Luis Bonaventura, (lead author, andre@selfchargebox.com) $)^{1,2}$ Rangel Graudiston Aredes, (rangel.aredes@gmail.com) ${ }^{1}$ Eduardo Antonelli, (antonelli@unifesp.br) ${ }^{1}$

${ }^{1}$ Advanced Ceramic Laboratory, Science and Technology Institute, Universidade Federal de São Paulo; ${ }^{2}$ Self Charge Box, StartUp, São José dos Campos - SP - Brazil. 